\title{
No Evidence of Metastatic Disease
}

National Cancer Institute

\section{Source}

National Cancer Institute. No Evidence of Metastatic Disease. NCI Thesaurus. Code C48558.

Diagnostic tests fail to detect presence of metastatic lesions. 